\title{
Confusion and representational practices as factors that sustain rich pedagogical discussions within faculty online learning communities
}

\author{
Chandra Turpen, ${ }^{1}$ Fred Goldberg, ${ }^{2}$ Adriana Corrales, ${ }^{3}$ and Ed Price ${ }^{4}$ \\ ${ }^{1}$ Univ. of Maryland, Dept. of Phys., Toll Physics Building, College Park, MD 20742 \\ ${ }^{2}$ San Diego State Univ., Dept. of Phys., 5500 Campanile Dr. San Diego, CA 92182 \\ ${ }^{3}$ San Diego State Univ., Center for Research in Mathematics and Science Education, 6475 Alvarado Rd, Suite 128 San Diego, CA 92120 \\ ${ }^{4}$ California State Univ. San Marcos, Dept. of Phys, 333 South Twin Oaks Valley Rd, San Marcos, CA 92096
}

\begin{abstract}
We present analysis of an exemplary case of rich and extended pedagogical reasoning about energy representations among physics faculty participating in the Next Generation Physical Science and Everyday Thinking Faculty Online Learning Community (FOLC). Our analysis is driven by the question, "What drives and sustains this prolonged and substantive pedagogical discussion?" We argue that this discussion was partially sustained through two factors: (1) the substantive confusion (for students and educators) that it generated and (2) the rich coordination of conceptual resources it involved. Some of this rich coordination of conceptual resources seems enabled by the common curriculum used among the educators in this FOLC. We suggest that these factors create opportunities for faculty to deepen their pedagogical content knowledge and critical reflectiveness.
\end{abstract}

\section{INTRODUCTION}

The Next Generation Physical Science and Everyday Thinking curriculum (NGPET) [1] is a guided-inquiry curriculum for preservice elementary teachers. NGPET was adapted from the Physics and Everyday Thinking (PET) suite [2-4], and is aligned with the Next Generation Science Standards. For flexible use, NGPET is modular and has parallel versions for studio or lecture classrooms [1].

Guided by the research on educational transformation and the dissemination of research-based instructional strategies $[5,6]$, we designed a faculty online learning community (FOLC) where faculty can support and learn from each other in their implementation of NGPET (http://ngpfolc.com). A long-term goal of the NGPET FOLC is to enhance faculty's pedagogical content knowledge and critical reflectiveness around issues of teaching and learning.

In the first full year ('17-'18) of the NGPET FOLC, there were 48 active members organized into four clusters, each with two or three cluster leaders (CLs) and eight to ten other members. CLs have extensive experience with PET and are knowledgeable about issues of teaching and learning. Other cluster members had varied backgrounds, from extensive experience with PET to no experience. All participants were expected to use the NGPET curriculum at least once per year during the project, providing a shared context for conversation. Each cluster met via Zoom video conferencing every other week and communicated asynchronously using an online platform. All video conferences were recorded, and project staff monitored two of the clusters in detail.

The synchronous cluster meetings provide a forum for faculty to discuss pedagogical, logistical or other issues and to learn from each other. The meetings lasted about one hour and it was common practice for faculty participants to take turns sharing issues with the group and getting comments or feedback in return. The conversations around any particular issue might typically last from a couple of minutes to ten minutes, rarely much longer. However, one session from a studio- style cluster included over 32 minutes of substantive pedagogical discussion around an issue raised by a cluster member. We examine this particular conversation in detail to identify factors that seemed to sustain it. There were three motivations for this analysis: first, from a Vygotskian perspective [7], faculty's interactions with each other and physical tools can serve as mediators of thought, and should be considered an integral part of their learning; second, an analysis of such a long conversation could help us understand how the conversation may promote pedagogical content knowledge and critical reflectiveness; third, insights about how to promote pedagogically rich discussions could be useful to our own FOLC and other FOLCs. These reasons are important and will be more fully addressed in future papers. In this paper, we focus our attention on addressing the research question: What factors seemed to drive and sustain this unusually long conversation?

\section{BACKGROUND ON HOW NGPET AND PET CURRICULA DESCRIBE ENERGY}

NGPET includes two energy representations (Fig. 1). An energy giver/energy receiver representation (G/R energy diagram) follows the flow of energy through a system of interacting objects. Figure 1(a) shows a G/R diagram for a person pushing a cart to increase its speed (and assuming negligible friction). During the interaction, energy is transferred from the giver (person) to the receiver (cart), there is a decrease in chemical potential energy (CPE) and an increase in kinetic energy (KE) in the giver, and an increase in kinetic energy in the receiver. Conservation of energy requires that the net decrease in energy in the giver (CPE - KE) equals the increase in KE in the receiver. Students are briefly introduced to a second representation, a systems approach where one chooses which objects are to be included in a system, and then considers the energy input to, and output from, the system, and energy changes within the system. Figure 1(b) shows a systems diagram with both the person's hand and the cart in the same system. In that case, there are no energy inputs or out- 


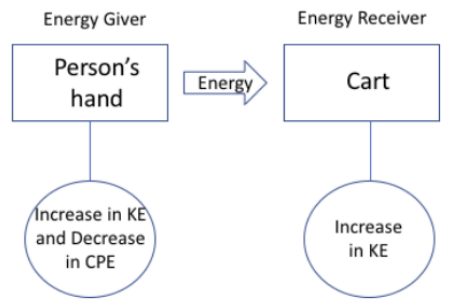

Fig. 1a

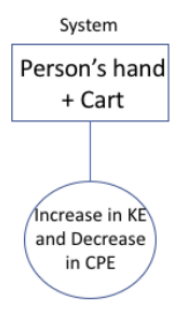

Fig. 1b
FIG. 1. Giver/receiver (G/R) (1a) and systems approach (1b) energy diagrams describing the interaction between a person's hand and a low-friction cart during the time the hand and cart are speeding up.

puts, but only energy changes. By conservation of energy, the energy changes equal zero. In NGPET, students describe elastic, magnetic, electrostatic and gravitational interactions using the G/R approach exclusively. Elastic, magnetic, electrostatic or gravitational potential energies can change, and magnetic, electrostatic or gravitational fields can be energy sources or receivers. In contrast, in the original version of PET students use the systems approach exclusively when describing magnetic, electrostatic and gravitational interactions.

\section{METHODS AND ANALYTICAL APPROACH}

Orienting Assumptions. Drawing on a Vygotskian perspective [7], we see learning as the bidirectional coordination between informal and formal knowledge. This coordination first occurs on a public, interpersonal plane, before understandings are internalized by learners. This perspective suggests that modeling the form and structure of distributed cognitive processes can give us insight into what faculty learn. Horn and colleagues [8-12] provide a conceptual bridge between Vygotsky and specific analytical constructs useful for understanding collaborative talk between educators.

Setting and Data Selection. This cluster meeting was chosen for its particularly long and pedagogically engaging discussion. There were 3 CLs (Carter, Courtney and Clay) and 4 members present and participating (Yin, and three others not speaking in the excerpt below). Carter and Courtney both had 10+ years of experience teaching the PET curriculum, but had only taught NGPET once before the current term. Yin had no experience teaching either PET or NGPET prior to this term. These three were all teaching NGPET in the studio-style format at the time. The focal meeting took place in Oct. 2017 and lasted for 1-hour. The 32-min. discussion we analyze began when Yin introduced a problem from her class.

Analytical Approach. To help understand what drove and sustained it, we divided the whole discussion into smaller segments representing slight shifts in the focus of the conversation. We then identified features of these segments of conversation that seemed common across all or most of the entire 32-minute discussion and proposed these as major factors in driving and sustaining it. We illustrate this process in Section
IV, where we present and analyze the transcript from one of these segments that occurred early in the entire conversation.

As we analyzed this conversation to determine what factors seemed to drive and sustain it, we drew on analytical constructs from Ilana Horn and colleagues [11] to hone our attention to particular "conceptual resources" [13] that instructors use in conversation: representations (e.g. artifacts, verbal or physical, that stand in for larger phenomena, which can be formal or informal) and representational practices (e.g. the ways in which people use artifacts to interpret the world). Other aspects of Horn's conceptual resources framework will be examined in future work. Our analysis illustrates the representations and representational practices that physics instructors used to puzzle through how to organize teaching and learning of the cross-cutting concept of energy.

\section{ANALYSIS}

Yin initiates this conversation by sharing how her students did not have trouble using the giver/receiver $(\mathrm{G} / \mathrm{R})$ energy diagram approach to describe interactions between two or more objects, or even for those involving magnetic, electrostatic and gravitational energy. They also did not have difficulty learning the systems approach. However, when Yin tried to introduce the systems approach as a second way of describing gravitational interactions (the curriculum only used the G/R approach), both she and her students got confused. She asks the cluster about this and whether it would be appropriate to use both approaches. One CL admits that he gets confused about the same thing and talks about some difficulties he has teaching both approaches. Spurred by a question from another faculty participant that had yet to teach with the NGPET curriculum, the CLs describe some of the differences between how PET and NGPET treat energy. This discussion reveals that for some of the educators highly experienced in teaching with PET, some of their confusion is arising from significant changes in how energy representations are introduced within the flow of activities across the two versions of curriculum. Importantly, this segment of the conversation establishes distinctions between the G/R and systems approaches to conservation of energy (as described in Section II). The segment we analyze here begins with Yin redefining her problem and contesting an explanation for students' confusion about systems posed by CL, Clay, near the end of the previous segment. Carter replies and generates a physical representation in response (Fig. 2). Table I shows the continuous transcript of this segment of discussion.

This exchange begins with Yin reanimating the kinds of things that she hears her students say and the kinds of things that she, as the teacher, conveys to her students in the classroom. This is a teaching replay [10, 12], reconstituting lived experiences from the classroom in a detailed storytelling mode. Within this storytelling, Yin describes her students' confusion in detail and vulnerably admits her own confusion. In the process, she refers to some specific activities within the NGPET curriculum as well as formal energy representa- 
TABLE I. Transcript of a continuous 2-minute segment of discussion between CLs, Courtney and Carter, and participant, Yin.

T1 Yin: Well, I think my activity seven (introducing the systems approach) actually went well. It wasn't too hard. It was defining the system. It's like, when we go to the field. Because in the past, the system is, "Okay, you had to include the person, or you don't include the person." (Fig. la and $1 b$ ) Now it's like, "Okay, now it's the field." If I say, okay, I should say, "Okay, include the earth." But then they (the students) are thinking, "There's a third thing called the field? What's going on?" I just don't really know, in terms of defining the system, like, it's not just the Earth. It's an extra field. Because the Earth and the field both appear in the $(G / R)$ energy diagram earlier. So that really makes it confusing...

T2 Carter: Yeah, I struggle with that. So, here's my - if you can see that. Here's my understanding, and I think this applies to both PET and Next Gen PET. (generates Fig. 2 above on a whiteboard)

T3 Courtney: Not really in PET. It is the Earth and the object system and then you talk about a decrease in potential energy and an increase in kinetic energy.

T4 Carter: I think if you wanted to do that you'd have to include the field in this box (pointing to the 'pen+earth' box).

T5 Courtney: Yes. (Yin shakes her head up and down.)

T6 Carter: So, you'd be moving this-

T7 Courtney: box to that box. Right. (Carter gestures moving 'field' box over to 'pen+earth' box.)

T8 Carter: So, that would be fine but to talk about a change in GPE, you have to be talking about the field (points to field box), whether the field is its own system or is part of a compound system (points to other box) that includes objects. Either way is okay. But I've-

T9 Yin: The field has to include objects, right? Without the (unintelligible) ball, and Earth, there is no ... I don't know (laughs).

T10 Carter: That's true. It's true that, um, but we're in charge of focusing our attention on whichever system we want, so I think there are places in the curriculum where the field, (points towards the 'field' box), whether it be a gravitational or a magnetic or an electrostatic field, is put by itself in a box and is thought of as an energy giver or receiver.

T11 Yin: Right... So, if I want to talk about different systems right here, I need to include field along with the Earth and the ball and the pen? T12 Carter: Yeah, I don't know. Maybe I'm wrong.

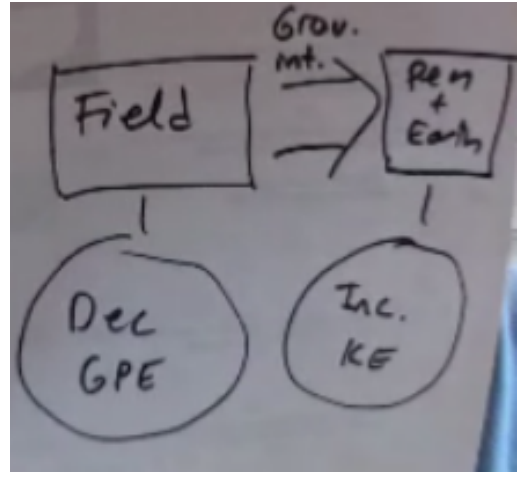

FIG. 2. Screenshot of a G/R diagram Carter generated.

tions within the NGPET curriculum. Then, in T2 we see CL Carter first normalize this confusion for himself as an educator and then draw an energy diagram for a falling pen that he thinks applies to both the original PET and NGPET (Fig. 2). In T3, Courtney makes a bid to modify the diagram that Carter has shared to make it more consistent with the original PET curriculum. Courtney's modification would revert the representation to a single system with no energy input or output, just two energy changes associated with the whole earth+pen+field system - an increase in kinetic energy and a decrease in potential energy (akin to Fig. 1b).

The participants then discuss whether this is a sensible way to describe the physical phenomenon, and alternatives are suggested. In the process, the purpose of and meanings associated with these representations are contested. Carter begins to revoice Courtney's suggestion (T4) and they proceed to finish each other's sentences as Carter coordinates their talk by gesturing to appropriate features in the whiteboard representation shown in Figure 2 (T5-T8). Yin tries to clarify her understanding of the imagined systems diagram, but admits she is still uncertain (T9). In T10, Carter reminds the group that "we're in charge of focusing our attention on whichever system we want" - conveying that they get to use these representational tools agentically in whatever ways are useful to them (in some ways contesting external authority possibly from curriculum or curriculum writers to represent things in one way). Carter asks the group to consider whether it would also be reasonable at times to consider the field to be an object in of itself acting as an energy giver or receiver to other systems. Yin again seeks clarification, but her question suggests she is still not sure. Lastly (T12), we see Carter is unsure about these different approaches.

Synthesis of Findings Throughout the specific segment presented, we see highly collaborative sensemaking occurring in this virtual setting with multiple speakers coordinating their talk and gestures around a representational artifact that Carter has generated in conversation. We see educators engaging in a deep discussion about the structure of the content, but not losing track of the curricular structures or students' engagement with these ideas. This substantive focus is representative of the 32 minute conversation. At one point, a little more than halfway through the long conversation, one of the cluster leaders suggests the group might move on to a different 
topic entirely, but because the other two CLs will be teaching the gravitational activity within the next week, and because Yin is still unsure how to help her students understand the systems approach, the group decides to continue their conversation, and does so for about fifteen additional minutes. The conversational features of collaborative sensemaking and rich representational use continue over many segments of talk of this 15-min. continuation (still in relation to these energy representations and Yin's classroom). We argue that this discussion was partially sustained through the substantive confusion (for students and educators) that it generated and the rich coordination of conceptual resources it involved. This detailed discussion also seems partially facilitated by the shared curricular context of our FOLC. The nature of the pedagogical problem that Yin presents focuses the group's attention on specific student confusions and results in confusion from the educators themselves. By confusion, we refer to the confusion expressed by Yin and the cluster leaders about their own understanding and their students' understanding of the physics content underlying the energy representations. Many of the teaching replays (from multiple faculty participants) recall student confusion about the systems approach and comfort with the G/R approach. For the FOLC participants, the issue Yin raises was not trivial. For the educators, there was confusion around: (1) How to decide which objects to include in the system when using the systems approach; (2) How to deal with the fact that an object cannot interact with the field that it generates itself. For faculty experienced with PET, there were additional confusions regarding changes in how energy was treated in the NGPET curriculum. These confusions all seem to have motivated participants to engage in substantive conversation.

A second critical factor was the rich use of conceptual representations by Yin and the CLs, referring to their use of both informal (classroom replays) and formal (energy diagrams) representations centered around their shared use of the same curriculum. When Carter drew his diagram (Fig. 2), he used it not only to describe a typical G/R energy diagram, but with appropriate gesturing he described how it could be morphed into a system-type energy diagram. Others participated in the discussion, with Carter's gestures serving as the vehicle for collaborative interpretation of the actual and imagined dia- grams. The diagram also provided the referent through which Carter, Yin and others could make explicit connections with their students' understandings and confusions. In addition to the use of both formal and informal representations shown here, the cluster members used them throughout the entire 32minute conversation. Later, Carter drew a systems diagram to describe a new problem scenario and another cluster leader produced the G/R diagram. Both diagrams provided a context for an extended discussion addressing some of the confusions mentioned previously. The CLs also displayed formal diagrams from the printed curriculum to illustrate the points they were trying to make. In summary, the extensive use of both the formal and informal representations helped drive and sustain the pedagogically rich conversation. We believe that additional factors contributed to sustaining this conversation (e.g. the immediacy of the problem for other educators' practical work, the group's conversational norms and questioning practices, the collective maintenance of agency with the problem "poser") which we intend to explore in future work.

\section{CONCLUSIONS}

Across the country, faculty learning communities are being organized around a wide range of topics/issues and operating with widely varying conversational routines. More detailed analyses of these professional development spaces are needed in order for us to more deeply understand what forms of learning may be supported by them. Our findings suggest that deeply engaging, productive conversations can take place when faculty grapple with issues that create substantive confusion, and involve rich coordination of conceptual resources. It may be useful to designers and facilitators of other FOLCs to cultivate such opportunities. In future work, we examine how these conversational structures contribute to developing pedagogical content knowledge and critical reflectiveness.

\section{ACKNOWLEDGMENTS}

We thank the faculty members of the NGPET FOLC that participated in this research study as well as Meghan Clemons, Alexandra Lau, and Melissa Dancy for feedback on our initial analysis. This work is supported by NSF-1626496.
[1] https://nextgenpet.activatelearning.com. Retrieved 7/6/18.

[2] F. Goldberg, S. Robinson, and V. Otero, Physics and Everyday Thinking. (Activate Learning, Greenwich CT, 2007).

[3] F. Goldberg, V. Otero, and S. Robinson, Am. J. Phys. 78, 1265 (2010).

[4] F. Goldberg, E. Price, S. Robinson, D. Boyd-Harlow, and M. McKean. Phys. Rev. ST Phys. Educ. Res. 8, 010121 (2012).

[5] C. Henderson, A. Beach, and N. Finkelstein, J. Res. Sci. Teach. 48, 952-984 (2011)

[6] C. Henderson, M. Dancy, and M. Niewiadomska-Bugaj, Phys. Rev. ST Phys. Educ. Res. 8, p. 020104 (2012).
[7] L. S. Vygotsky, Mind in society (Harvard Univ., Cambridge, 1980).

[8] I. S. Horn (2005). Cognition and Instruction, 23, 181-217.

[9] I. S. Horn (2007). J. Learn. Sci., 16, 37-79.

[10] I. S. Horn (2010). The Teachers College Record, 112, 225-259.

[11] I. S. Horn and B. D. Kane (2015). J. Learn. Sci., 24, 373-418.

[12] I. S. Horn and J. W. Little, Am. Educ. Res. J. 47, 181-217 (2010).

[13] We caution readers that Horn et al's definition is different from the Resources or Knowledge-In-Pieces frameworks (from A. diSessa, D. Hammer, E. Redish, and A. Elby) common in PER. 